PROCEEDINGS OF THE

AMERICAN MATHEMATICAL SOCIETY

Volume 130, Number 10, Pages 2995-2998

S 0002-9939(02)06482-1

Article electronically published on March 29, 2002

\title{
AN EXAMPLE OF AMENABLE KAC SYSTEMS
}

\author{
CHI-KEUNG NG
}

(Communicated by David R. Larson)

\begin{abstract}
By giving an interesting characterisation of amenable multiplicative unitaries, we show, in a very simple way, that bicrossproducts of amenable locally compact groups are both amenable and coamenable.
\end{abstract}

The study of amenability of Kac algebras began with [6]. In [2], amenability of regular multiplicative unitaries was also defined. Recently, we studied in 9] and [11] amenable Hopf $C^{*}$-algebras and amenable Kac algebras. However, there has not been any non-trivial example of amenable Kac algebra (or Kac system) explicitly stated in any literature so far (at the same time that this work was finished, we learned that E. Bedos, G.J. Murphy and L. Tuset have recently proved in [4] that the compact quantum groups $S U_{q}(2)$ is coamenable; moreover, they also have a version of Proposition 3 for compact quantum groups although their proof of $S U_{q}(2)$ being coamenable does not require it).

In this note, we will give a simple proof for the following statement: in the construction of the "bicrossproduct" of two locally compact groups (in [3, §1]), if one of the groups is amenable, then the bicrossproduct is either amenable or coamenable (depending on the convention). This statement does not appear in any literature so far. Note that as the "reduced algebra" of the bicrossproduct is the reduced crossed product, one could imagine that the "full algebra" is the full crossed product but it seems not an obvious fact (and to prove the above through this route seems to be more complicated than the treatment here). Our proof for the above statement uses a simple but seemingly powerful characterisation (as the condition is relatively easy to check) for amenable $C^{*}$-multiplicative unitaries (Proposition 3).

Let us first recall from [8, $2.1 \& 2.2(\mathrm{~b})]$ as well as [2, A.13(c)] the following notations.

Definition 1. (a) Let $V$ be a multiplicative unitary on $H$ (in the sense of 2 1.1]). Then $V$ is called a $C^{*}$-multiplicative unitary if for any representation $X$ and co-representation $Y$ of $V$ (see [2, A.1]), the sets

$$
\hat{S}_{X}=\overline{\left\{(\mathrm{id} \otimes \omega)(X): \omega \in \mathcal{L}(H)_{*}\right\}}
$$

and

$$
S_{Y}=\overline{\left\{(\omega \otimes \mathrm{id})(Y): \omega \in \mathcal{L}(H)_{*}\right\}}
$$

Received by the editors January 3, 2001 and, in revised form, May 9, 2001.

2000 Mathematics Subject Classification. Primary 46L05, 46L55; Secondary 43A07, 22D25.

(C)2002 American Mathematical Society 
are $C^{*}$-algebras such that $X \in M\left(\hat{S}_{X} \otimes S_{V}\right)$ and $Y \in M\left(\hat{S}_{V} \otimes S_{Y}\right)$. (Recall that in this case, $S_{V}$ and $\hat{S}_{V}$ are Hopf $C^{*}$-algebras with coproducts $\delta$ and $\hat{\delta}$ defined by the formulas in [2 3.8$]$.)

(b) Let $V$ be a $C^{*}$-multiplicative unitary. For any Hilbert space $K$, a unitary $U \in M\left(\mathcal{K}(K) \otimes S_{V}\right)$ is called a unitary corepresentation if

$$
(\mathrm{id} \otimes \delta)(U)=U_{12} U_{13}
$$

(recall that unitary corepresentations of $S_{V}$ correspond bijectively to representations of $V)$. Moreover, there exists a universal object $\left(\hat{S}_{p}, V^{\prime}\right)$ for unitary corepresentations of $S_{V}$ in the sense that $\hat{S}_{p}$ is a $C^{*}$-algebra and $V^{\prime} \in M\left(\hat{S}_{p} \otimes S_{V}\right)$ is a unitary satisfying $($ id $\otimes \delta)\left(V^{\prime}\right)=V_{12}^{\prime} V_{13}^{\prime}$ such that for any unitary corepresentation $U$ of $S_{V}$, there exists a canonical surjective $*$-homomorphism $\pi_{U}$ from $\hat{S}_{p}$ to $\hat{S}_{U}$ with $U=\left(\pi_{U} \otimes\right.$ id $)\left(V^{\prime}\right)$.

(c) A $C^{*}$-multiplicative unitary $V$ is said to be amenable if the trivial representation $\pi_{1}$ (i.e. the representation corresponding to the trivial unitary corepresentation $\left.1 \in M\left(S_{V}\right)\right)$ is weakly contained in $\pi_{V}$. On the other hand, $V$ is called coamenable if $\Sigma V^{*} \Sigma$ is amenable (where $\Sigma(\zeta \otimes \eta)=\eta \otimes \zeta$ ).

Any regular ([2]) or semi-regular and équilibré ([1]) or manageable (13]) multiplicative unitary is a $C^{*}$-multiplicative unitary. Note that part (c) is only one of the many equivalent formulations for amenability (see e.g. [9] for some of these equivalences; see also [11] for more of them in the case of Kac algebras).

To prove the main theorem, we need Proposition 3 below which is a partial generalisation of [11,5.1(XII)] (in the sense that we consider only the one-dimensional representations instead of the finite-dimensional ones). The proof of this proposition requires the following lemma. The lemma itself follows from [10, 5.5] (note that if $L$ is the canonical representation of $S$ on $\mathcal{L}(H)$, then $\left(L, \pi_{V}\right)$ is a $V^{\prime}$-covariant representation in the sense of [10,4.1]).

Lemma 2. Let $V$ be a $C^{*}$-multiplicative unitary on a Hilbert space $H$. Suppose that $\tilde{\pi}_{V}$ is the extension of $\pi_{V}$ to $\hat{S}_{p}^{* *}$ (considered as a representation on $H$ ) and $e$ is the support projection of $\tilde{\pi}_{V}$. Then $\hat{\delta}_{p}^{* *}(e) \geq e \otimes 1$ (recall that $\hat{S}_{p}$ is a Hopf $C^{*}$-algebra with a coproduct $\hat{\delta}_{p}$ ).

Proposition 3. Suppose that $V$ is a $C^{*}$-multiplicative unitary on $H$. Then $V$ is amenable if and only if $\hat{S}_{V}$ has a one-dimensional representation.

Proof. Since the amenability of $V$ will imply the existence of a counit for $\hat{S}_{V}$ (see e.g. $[9,3.5]$ ), we need only to show the converse. Suppose that $\psi$ is a one-dimensional representation of $\hat{S}_{V}$. Then $U=(\psi \otimes \mathrm{id})(V) \in M\left(S_{V}\right)$ is a unitary corepresentation of $S_{V}$. This means that $U$ is a group-like element in $M\left(S_{V}\right)$ and $\pi_{U}=\psi \circ \pi_{V}$. Moreover, $U^{*}$ is again a group-like unitary and hence a unitary corepresentation of $S_{V}$ on $\mathbb{C}$ (or equivalently, a representation of $V$ on $\mathbb{C}$ ). Now $\left(\psi \circ \pi_{V} \otimes \pi_{U^{*}}\right) \circ \hat{\delta}_{p}=$ $\left(\pi_{U} \otimes \pi_{U^{*}}\right) \circ \hat{\delta}_{p}=\pi_{U U^{*}}=\pi_{1}$. Using the argument of [11, 3.6], we see that $\operatorname{ker} \pi_{V} \subseteq \operatorname{ker}\left(\pi_{V} \otimes \mathrm{id}\right) \circ \hat{\delta}_{p}$. For the benefit of the readers, we will repeat the simple argument here. Note that ker $\pi_{V}=(1-e) \hat{S}_{p}^{* *} \cap \hat{S}_{p}$ (where $e$ is the support projection of $\tilde{\pi}_{V}$ as in Lemma 2) and so $x=(1-e) x$ for any $x \in \operatorname{ker} \pi_{V}$. This, together with 
$(e \otimes 1) \hat{\delta}_{p}^{* *}(1-e)=0$ (because of Lemma 2), implies that

$$
\begin{aligned}
\left(\pi_{V} \otimes \mathrm{id}\right) \hat{\delta}_{p}(x) & =\left(\tilde{\pi}_{V} \otimes \mathrm{id}\right) \hat{\delta}_{p}^{* *}(x)=\left(\tilde{\pi}_{V} \otimes \mathrm{id}\right) \hat{\delta}_{p}^{* *}((1-e) x) \\
& =\left(\tilde{\pi}_{V} \otimes \mathrm{id}\right)\left((e \otimes 1) \hat{\delta}_{p}^{* *}((1-e) x)\right)=0 .
\end{aligned}
$$

Hence $\operatorname{ker} \pi_{V} \subseteq \operatorname{ker}\left(\psi \otimes \pi_{U^{*}}\right) \circ\left(\pi_{V} \otimes \mathrm{id}\right) \circ \hat{\delta}_{p}=\operatorname{ker} \pi_{1}$. In other words, $\pi_{1}$ is weakly contained in $\pi_{V}$.

Remark 4. In the case of Kac algebras, we can obtain the above by an extremely simple proof because $\pi_{U^{*}}=\psi \circ \hat{\kappa} \circ \pi_{V}$ and we do not need Lemma 2,

Moreover, we have the following interesting consequences of this proposition. Part (b) of the corollary is a generalisation of [4, 2.10] and is an inferior version of 11, 5.3(b)]. We were told that [11, 5.3(b)] (and hence part (b) below) is already known although it is not found in any literature.

Corollary 5. (a) Let $V$ and $W$ be two $C^{*}$-multiplicative unitaries. If $V$ is amenable and $\hat{S}_{V}$ is isomorphic to $\hat{S}_{W}$ as $C^{*}$-algebras, then $W$ is also amenable.

(b) A locally compact group $G$ is amenable if and only if $C_{r}^{*}(G)$ has a onedimensional representation.

As another application of the above proposition, we obtain our main theorem below. From now on, $G$ is a locally compact group and $G_{1}$ and $G_{2}$ are two closed subgroups of $G$ such that the map $\varphi: G_{1} \times G_{2} \rightarrow G$ given by $\varphi(r, g)=r g$ is a homeomorphism from $G_{1} \times G_{2}$ onto an open dense subset $\Omega$ of $G$ (see [3, §1]). Consider the canonical actions $\alpha$ and $\beta$ of $G_{1}$ and $G_{2}$ on $G_{1} \backslash G$ and $G / G_{2}$ respectively. Note that both $\alpha$ and $\beta$ act by automorphisms. Let $V$ be the semi-regular irreducible multiplicative unitary as in [3, 1.1]. Note that $V$ is a $C^{*}$-multiplicative unitary as irreducible multiplicative unitary is équilibré.

Theorem 6. With the notation as above, if $G_{1}$ (respectively, $G_{2}$ ) is amenable, then $V$ is amenable (respectively, coamenable).

Proof. By [3, 1.1(c)], we know that $\hat{S}_{V}$ is isomorphic to the reduced crossed product $C_{0}\left(G_{1} \backslash G\right) \times_{\alpha, r} G_{1}$. Therefore, using Proposition 3 it suffices to show that $C_{0}\left(G_{1} \backslash G\right) \times{ }_{\alpha, r} G_{1}$ has a one-dimensional representation. Let $\phi$ be the one-dimensional representation on $C_{0}\left(G_{1} \backslash G\right)$ given by the evaluation at the coset $[e]=G_{1}$. Let $\pi$ be the trivial representation of $G_{1}$ (in fact, we can take any one-dimensional representation). We note that $(\phi, \pi)$ is a covariant representation for $\alpha$. Indeed, for any $f \in C_{0}\left(G_{1} \backslash G\right)$, we have $\phi\left(\alpha_{s}(f)\right)=f\left(\alpha_{s^{-1}}([e])\right)=f([e])=\phi(f)=\pi(s) \phi(f) \pi(s)^{*}$. Hence, we obtained a one-dimensional representation for the full crossed product $C_{0}\left(G_{1} \backslash G\right) \times_{\alpha, \max } G_{1}$ which is identical with the reduced crossed product $C_{0}\left(G_{1} \backslash G\right) \times{ }_{\alpha, \mathrm{r}} G_{1}$ (as $G_{1}$ is amenable).

Note that $\Omega=G$ if and only if $G_{1} \cong G / G_{2}$ and $G_{2} \cong G_{1} \backslash G$ under the canonical maps. In this case, the actions $\alpha$ and $\beta$ are given by the commutation rule $g r=$ $\beta_{g}(r) \alpha_{r}(g)$ (that comes from the bijectivity of $\varphi$; see [2, $\left.\S 8\right]$ ). Moreover, $\alpha$ and $\beta$ satisfy the conditions for matching pairs in 7]. (except those concerning the RadonNikodym derivatives). Recall that in this case, $G$ is the bicrossproduct group as defined in [12, $\S 2]$. Note that the bicrossproduct group can only be defined for a matching pair with $\alpha_{e}$ and $\beta_{e}$ being the identity maps (or equivalently, $\alpha$ and $\beta$ act by automorphisms). 
On the other hand, in this case (i.e. $\Omega=G),\left(L^{2}(G), V, U\right)$ (where $U$ is the unitary as in [3, 1.1(b)]) is the Kac system bicrossproduct of $\left(L^{2}\left(G_{1}\right), X, u\right)$ (the canonical Kac system of $G_{1}$ as given in [2] 6.11(b)]) and $\left(L^{2}\left(G_{2}\right), Y, v\right)$ relative to $Z$ (see [2, 8.13, $8.15 \& 8.21]$ ). Therefore, Theorem [6 applies to Kac system bicrossproducts of locally compact groups (as defined in [2, §8]). If, in addition, $\alpha$ and $\beta$ in the above satisfy the conditions for the Radon-Nikodym derivatives in [7, this Kac system actually comes from a Kac algebra which will be amenable and coamenable if both $G_{1}$ and $G_{2}$ are amenable.

\section{REFERENCES}

1. S. Baaj, Représentation régulière du groupe quantique des déplacements de Woronowicz, Astérisque (1995), no. 232, 11-48. MR 97a:46107

2. S. Baaj and G. Skandalis, Unitaires multiplicatifs et dualité pour les produits croisés de $C^{*}$ algèbres, Ann. scient. Éc. Norm. Sup., $4^{e}$ série, t. 26 (1993), 425-488. MR 94e:46127

3. S. Baaj and G. Skandalis, Transformations pentagonales, C. R. Acad. Sci. Paris Sr. I Math. 327 (1998), no. 7, 623-628. MR 99k:28018

4. E. Bédos, G.J. Murphy and L. Tuset, Co-Amenability of Compact Quantum Groups, J. Geom. Phys. 40 (2001), 130-153. CMP 2002:03

5. M.E.B. Bekka, Amenable unitary reperesentations of locally compact groups, Invent. Math. 100 (1990), 383-401. MR 91g:22007

6. M. Enock and J.-M. Schwartz, Algebres de Kac moyennables, Pacific J. Math. 125 (1986), 363-379. MR 88f:46126

7. S. Majid, Hopf von Neumann algebra bicrossproducts, Kac algebra bicrossproducts and the classical Yang-Baxter equations, J. Funct. Anal. 95 (1991), 291-319. MR 92b:46088

8. C.K. Ng, Morphisms of Multiplicative unitaries, J. Oper. Theory 38 (1997), 203-224. MR 99i: 46044

9. C.K. Ng, Amenability of Hopf $C^{*}$-algebras, Proceedings of the 17th OT conference (2000), 269-284. MR 2001g:46129

10. C.K. $\mathrm{Ng}$, Duality of Hopf $C^{*}$-algebras, preprint.

11. C.K. Ng, Amenable representations and Reiter's property for Kac algebras, preprint.

12. T. Yamanouchi, Bicrossproduct Kac algebras, Bicrossproduct groups and von Neumann algebras of Takesaki's type, Math. Scand. 71 (1992), 252-260. MR 94f:46097

13. S. L. Woronowicz, From multiplicative unitaries to quantum groups, Internat. J. Math. 7 (1996), no.1, 127-149. MR 96k:46136

Department of Pure Mathematics, The Queen's University of Belfast, Belfast BT7 1 NN, United Kingdom

E-mail address: c.k.ng@qub.ac.uk 